PREPARED FOR THE U.S. DEPARTMENT OF ENERGY, UNDER CONTRACT DE-AC02-76CH03073

PPPL-3537

PPPL-3537

UC-70

Nonlinear Charge and Current Neutralization

of an Ion Beam Pulse in a Pre-formed Plasma

by

Igor D. Kaganovich, Gennady Shvets,

Edward Startsev, and Ronald C. Davidson

January 2001

NM|

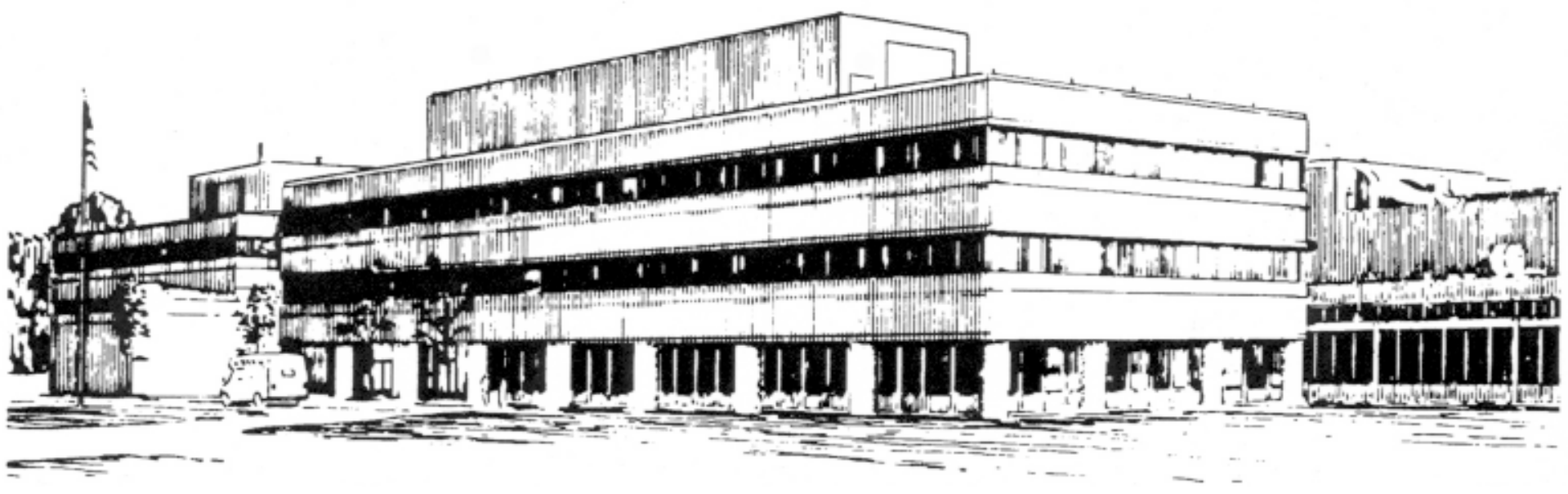

PRINCETON PLASMA PHYSICS LABORATORY PRINCETON UNIVERSITY, PRINCETON, NEW JERSEY 


\section{PPPL Reports Disclaimer}

This report was prepared as an account of work sponsored by an agency of the United States Government. Neither the United States Government nor any agency thereof, nor any of their employees, makes any warranty, express or implied, or assumes any legal liability or responsibility for the accuracy, completeness, or usefulness of any information, apparatus, product, or process disclosed, or represents that its use would not infringe privately owned rights. Reference herein to any specific commercial product, process, or service by trade name, trademark, manufacturer, or otherwise, does not necessarily constitute or imply its endorsement, recommendation, or favoring by the United States Government or any agency thereof. The views and opinions of authors expressed herein do not necessarily state or reflect those of the United States Government or any agency thereof.

\section{Availability}

This report is posted on the U.S. Department of Energy's Princeton Plasma Physics Laboratory Publications and Reports web site in Calendar Year 2001. The home page for PPPL Reports and Publications is: http://www.pppl.gov/pub_report/

DOE and DOE Contractors can obtain copies of this report from:

U.S. Department of Energy

Office of Scientific and Technical Information

DOE Technical Information Services (DTIS)

P.O. Box 62

Oak Ridge, TN 37831

Telephone: (865) 576-8401

Fax: (865) 576-5728

Email: reports@adonis.osti.gov

This report is available to the general public from:

National Technical Information Service

U.S. Department of Commerce

5285 Port Royal Road

Springfield, VA 22161

Telephone: 1-800-553-6847 or

(703) 605-6000

Fax: (703) 321-8547

Internet: http://www.ntis.gov/ordering.htm 


\title{
Nonlinear charge and current neutralization of an ion beam pulse in a pre-formed plasma
}

\author{
Igor D. Kaganovich, Gennady Shvets, Edward Startsev and Ronald C. Davidson \\ Plasma Physics Laboratory, Princeton University, Princeton, NJ 08543
}

(January 22, 2001)

The propagation of a high-current finite-length ion beam in a cold pre-formed plasma is investigated. The outcome of the calculation is the quantitative prediction of the degree of charge and current neutralization of the ion beam pulse by the background plasma. The electric and magnetic fields generated by the ion beam are studied analytically for the nonlinear case where the plasma density is comparable in size with the beam density. Particle-in-cell simulations and fluid calculations of current and charge neutralization have been performed for parameters relevant to heavy ion fusion assuming long, dense beams with length $l>>V_{b} / \omega_{b}$, where $V_{b}$ is the beam velocity, and $\omega_{b}$ is the electron plasma frequency evaluated with the ion beam density. An important conclusion is that for long, nonrelativistic ion beams, charge neutralization is, for all practical purposes, complete even for very tenuous background plasmas. As a result, the self-magnetic force dominates the electric force and the beam ions are always pinched during beam propagation in a background plasma.

\section{INTRODUCTION}

Understanding the transport of charged particle beams in background plasma is important for fundamental physics as well as for a variety of applications. As early as 1939 [1, 2], it was pointed out that the transport of cosmic rays may be governed by the charge and current neutralization by the ambient plasma. The recent resurgence of interest in charged particle beam transport in background plasma is brought about by the suggestion that the plasma can be used as a magnetic lens. Applications of the plasma lens range from heavy ion fusion to high energy lepton colliders [3-10]. In particular, both heavy ion fusion and high 
energy physics applications involve the transport of positive charges in plasma: partially stripped heavy elements for heavy ion fusion; positrons for electron-positrons colliders [9]; and high-density laser-produced proton beams for the fast ignition of inertial confinement fusion targets. The emphasis of the present work on positive ions is deliberate because, as we demonstrate below, the transport of positive ion beams through background plasma is very different from that of the negatively charged beams. A beam of positively charged particles attracts plasma electrons into the beam, whereas a beam of negative charges repels the electrons out of its path. An important consequence, which is one of the findings of the present calculation, is that a nonrelativistic positive ion beam with density $n_{b}$ can be neutralized to a very high degree by a large-volume tenuous plasma with ambient density $n_{p}<<n_{b}$

The beam charge and current neutralization by plasma electrons is an important issue for beam propagation in a background plasma. Beam focusing schemes rely on complete charge neutralization and partial current neutralization for magnetic focusing in plasma lenses [5], and for ballistic ion focusing in heavy ion fusion [8]. In these applications, the plasma is pre-formed by an external plasma source and is independent of the beam characteristics.

The goals of the present calculation are: (a) to derive a system of reduced equations for the electric and magnetic field generated by an ion beam propagating through background plasma, and (b) to develop a semi-analytical method for robust and easy assessment of the effects of these fields on the beam transport. The case where the beam propagates through a cold unmagnetized plasma, with plasma density large compared with the beam density, can be studied by use of linear perturbation theory $[3,4]$. The transport of relativistic electron beams was studied in detail in various contexts $[4,5,11]$. The transport of stripped pinched ion beam has been also discussed in [8], where the assumption of current neutrality was made to determine self-consistent solutions for the electric and magnetic fields. Here, we focus on the nonlinear case where the plasma density has an arbitrary value compared with the beam density, and correspondingly the degree of current neutralization is arbitrary. For simplicity, we neglect transient effects, and assume steady-state properties in the frame of 
the beam. Rosenbluth, et al. [12] have considered the equilibrium of an isolated, chargeneutralized, self-pinched ion beam pulse, in the absence of background plasma. In contrast, we consider the case where "fresh" uniform plasma is always available in front of the beam.

To simplify the analysis and make the problem tractable, a number of assumptions have been made. First, we neglect the dynamics of the beam ions and plasma ions. The beam ions are assumed to be moving in the $z$ direction with constant axial velocity $V_{b}$. The response time of the plasma ions is determined by the ion plasma frequency, which is much longer than the electron response time. Therefore, neglecting the dynamics of the plasma ions is well justified. Second, the entrance of the beam into the plasma will not be addressed in the present model. Furthermore, beam ionization effects are neglected, and the background plasma in front of the beam is assumed to be uniform and stationary. As a result, all field quantities (electric and magnetic), and the plasma and beam charge densities and current densities are stationary in a reference frame moving axially with the beam.

Additional simplifications of the electron fluid equations are possible for long beams where the beam half length $\left(l_{b}\right)$ is much longer than both the beam radius $\left(r_{b}\right)$ and the plasma neutralization length, which is equal to the ratio of the beam velocity $\left(V_{b}\right)$ to the electron plasma frequency $\left(\omega_{p}\right)$. The first assumption is used in the Darwin model [13]. The second assumption allows further simplifications. We show that under these conditions a reduction of the dimensionality of the problem is possible. For an axisymmetric beam, the longitudinal electron flow velocity is determined by a one-dimensional equation in the radial direction for each axial slice of the beam. Once the longitudinal electron flow velocity is determined, the electric and magnetic fields can be calculated from simple analytical expressions.

As an application of the theoretical model, we study transport of the ion beam pulse in the target chamber for heavy ion fusion. At the present time, the main approach to heavy ion fusion is ballistic focusing in the target chamber from an initial beam radius of about $3 \mathrm{~cm}$ down to a spot size of about $3 \mathrm{~mm}$. The beam traverses the chamber (radius about $3 \mathrm{~m}$ ), in near vacuum (a few mTorr of flibe vapor). Typical beam parameters are [8]: $C s^{+}$ions with 
energy $2.5 \mathrm{GeV}$, beam velocity $V_{b} \approx 0.2 c$, beam current $\approx 4 k A$, main pulse duration $10 \mathrm{~ns}$, and power in the pulse $\approx 3 M W$. The beam ion density ranges from $n_{b}=10^{11}-10^{13} \mathrm{~cm}^{-3}$, depending on the beam radius, providing space-charge potentials of a few $M V$. This large ion space charge is to be neutralized by a background plasma. The plasma can be created in the chamber by an external plasma source, by gas ionization by the beam ions, and by photoionization from the target, which is bombarded by beam ions preceding the main pulse. Both electrostatic defocusing and magnetic pinching of the beam have to be avoided for controlled ballistic focusing. Thus large self-electric and self-magnetic fields have to be avoided during focusing of the ion beam pulse. Since the beam parameters vary significantly for different heavy ion fusion scenarios, analytical results are of considerable importance for parametric studies, benchmarking of numerical codes, and comparison with experiments.

\section{BASIC EQUATIONS FOR DESCRIPTION OF ION BEAM PULSE PROPAGATION IN A PLASMA}

We consider all equations in the reference frame of the laboratory plasma, assuming that all quantities are stationary in the reference frame of the moving beam, i.e. all quantities depend on $t$ and $z$ exclusively through the variable $\zeta=V_{b} t-z$, which is the distance from the beam head. The ion response time is assumed to be large compared with the beam pulse duration, and, therefore, the background plasma ion density remains uniform during beam propagation. The beam density profile is also assumed to be given. The plasma electron density $n_{e}$, however, is a function of both the unperturbed density $n_{p}$, and the ion beam density profile. The plasma electrons are assumed to be cold, and electron thermal effects are neglected. This approach has been widely used to study laser-plasma interactions $[4$, 5, 11]. These assumptions are well justified for ion beam pulses envisioned for heavy ion fusion.

The electron fluid equations together with Maxwell's equations comprise a complete system of equations describing the electron response to a propagating ion beam pulse. The 
electron fluid equations consist of the continuity equation,

$$
\frac{\partial n_{e}}{\partial t}+\nabla \cdot\left(n_{e} \mathbf{V}_{e}\right)=0
$$

and the force balance equation,

$$
\frac{\partial \mathbf{p}_{e}}{\partial t}+\left(\mathbf{V}_{e} \cdot \nabla\right) \mathbf{p}_{e}=-e\left(\mathbf{E}+\frac{1}{c} \mathbf{V}_{e} \times \mathbf{B}\right)
$$

where $-e$ is the electron charge, $m$ is the electron rest mass, $\mathbf{V}_{e}$ is the electron flow velocity, $p=\gamma_{e} m \mathbf{V}_{e}$ is the electron momentum, and $\gamma_{e}=1 / \sqrt{1-V_{e}^{2} / c^{2}}$ is the relativistic mass factor. Maxwell's equations for the self-generated electric and magnetic fields, $\mathbf{E}$ and $\mathbf{B}$, are given by

$$
\begin{gathered}
\nabla \times \mathbf{B}=\frac{4 \pi e}{c}\left(Z_{b} n_{b} \mathbf{V}_{b}-n_{e} \mathbf{V}_{e}\right)+\frac{1}{c} \frac{\partial \mathbf{E}}{\partial t} \\
\nabla \times \mathbf{E}=-\frac{1}{c} \frac{\partial \mathbf{B}}{\partial t}
\end{gathered}
$$

where $\mathbf{V}_{b}$ is the ion beam velocity, $n_{e}$ and $n_{b}$ are the number densities of the plasma electrons and beam ions, respectively, and $Z_{b}$ is the ion beam charge state.

Considerable simplification can be achieved by applying the conservation of generalized vorticity. Indeed, operating on the electron momentum equation (2) with $\nabla \times$, and making use of Eq.(4), we obtain the equation for the generalized vorticity $\Omega=\nabla \times \mathbf{p}_{e}-e \mathbf{B} / c$, i.e.,

$$
\frac{\partial \boldsymbol{\Omega}}{\partial t}-\nabla \times\left(\mathbf{V}_{\mathbf{e}} \times \mathbf{\Omega}\right)=0
$$

The generalized vorticity $\Omega$ is equal to zero in front of the beam, and, therefore, it is equal to zero everywhere. Thus the magnetic field $\mathbf{B}$, is related to the electron flow velocity $\mathbf{V}_{e}$ by

$$
\mathbf{B}=\frac{c}{e} \nabla \times \mathbf{p}_{e}
$$

which has the form of the London equation for superconductivity [14]. Note that Eq.(6) is an exact result, and is not obtained under linearization assumptions. Making use of the London equation (6), the electron momentum equation (2) simplifies to become 


$$
\frac{\partial \mathbf{p}_{e}}{\partial t}+\nabla\left(K_{e}\right)=-e \mathbf{E}
$$

where $K_{e}=\gamma_{e} m c^{2}$ is the electron energy. For laser-plasma interactions, the hydrodynamic equations in this form were displayed in [14].

Note that the inertia terms in Eq. (2) are comparable in size to the Lorentz force term and cannot be omitted. Estimating the magnetic field from Eq.(11), one concludes that the electron gyroradius, $\rho_{e}=V_{e z} m c / e B$, is of order the beam radius. This is a consequence of the fact that the electrons originate from the region of zero magnetic field in front of the beam. If most electrons are dragged along with the beam and originate from the region of large magnetic field, the situation may be different [12].

\section{A. Electrostatic and vector potentials}

Various gauges are available to address the present problem. These include: the Coulomb gauge [4], the transverse Coulomb gauge, zero electrostatic potential [15], and even more complicated gauges designed to cancel specific terms in Maxwell's equations [3]. For com-

pletness, we note that representing $\mathbf{B}=\nabla \times \mathbf{A}$, and $\mathbf{E}=-\nabla \phi-\mathbf{c}^{-1} \partial \mathbf{A} / \partial t$, the form of Eq.(7) suggests that the natural choice of gauge is

$$
e \phi=K_{e},
$$

which automatically yields

$$
\frac{e}{c} \mathbf{A}=\mathbf{p}_{e}
$$

All other gauges require that the vector potential $\mathbf{A}$ would differ from the momentum $c \mathbf{p}_{e} / e$ by the gradient of an arbitrary function, which follows from (6). For example, in the present analysis, the full Coulomb gauge is not convenient because of the necessity to make the vector potential divergence free $\nabla \cdot \mathbf{A}=0$.

For cylindrically symmetric beams, it is convenient to use the transverse Coulomb gauge $\nabla_{\perp} \cdot \mathbf{A}=0$. In cylindrical geometry, assuming axisymmetry in the azimuthal direction, it 
follows that $\mathbf{A}_{\perp}=0$, and the fields are completely described by the electrostatic potential $\phi$, and the $z$-component of the vector potential $\mathbf{A}=A_{z} \widehat{\mathbf{e}_{z}}$, where $\widehat{\mathbf{e}_{z}}$ is a unit vector along the $z$-axis. Therefore, the fields can be expressed as

$$
\mathbf{B}=\nabla A_{z} \times \widehat{\mathbf{e}_{\mathbf{z}}}, \quad \mathbf{E}=-\nabla \phi-\frac{1}{c} \frac{\partial A_{z}}{\partial t} \widehat{\mathbf{e}_{\mathbf{z}}}
$$

Integration of the London equation (6) gives an explicit equation for the vector potential

$$
\frac{e}{c} A_{z} \widehat{\mathbf{e}_{\mathbf{z}}}=\mathbf{p}_{e}-\nabla \varkappa
$$

where $\varkappa$ is an unknown function. From the radial component of Eq.(11) it follows that

$$
\varkappa(\zeta, r)=-\int_{r}^{\infty} p_{e r} d r
$$

and the longitudinal component of the vector potential can be expressed as

$$
\frac{e}{c} A_{z}=p_{e z}+\frac{\partial \varkappa}{\partial \zeta}
$$

where $\zeta=V_{b} t-z$. Finally, the electrostatic potential $\phi$ can be determined by integrating the radial component of Eq.(7), which gives

$$
e \phi=K_{e}+V_{b} \frac{\partial \varkappa}{\partial \zeta}
$$

\section{APPROXIMATE SYSTEM OF EQUATIONS FOR LONG BEAMS}

In this section, an approximate set of equations is derived for a long, cylindrically symmetric beam satisfying

$$
l_{b}>>V_{b} / \omega_{p}, l_{b}>>r_{b},
$$

where $\omega_{p}=\left(4 \pi e^{2} n_{e} / m\right)^{1 / 2}$ is the electron plasma frequency. We also assume that the fields and electron flow velocity and density are in steady-state in the reference frame moving with the beam. The electron flow velocity is found by substituting Eq. (6) into the $\nabla \times \mathbf{B}$ Maxwell equation (3), which yields 


$$
-\frac{d}{r d r}\left[r\left(\frac{d p_{e z}}{d r}+\frac{\partial p_{e r}}{\partial \zeta}\right)\right]=\frac{4 \pi e^{2}}{c^{2}}\left(Z_{b} n_{b} V_{b}-n_{e} V_{e z}\right)+\frac{e V_{b}}{c^{2}} \frac{\partial E_{z}}{\partial \zeta}
$$

For long beams with $l_{b}>>V_{b} / \omega_{p}$, the displacement current [the final term on the righthand side of Eq. (14)] is of order $\left(V_{b} / \omega_{p} l_{b}\right)^{2}<<1$ compared with electron current. Because $l_{b}>r_{b}$ is assumed, the second term on the left-hand side of Eq. (14) is of order $\left(r_{b} / l_{b}\right)^{2}(<<1)$ smaller than the first term on the left-hand side. As we shall prove below, the electron flow velocity does not approach ultrarelativistic values even for $\beta_{b} \longrightarrow 1$, therefore, ultrarelativistic effects are not important.

For sufficiently long beams $\left(l_{b}>>V_{b} / \omega_{p}\right.$ and $\left.l_{b}>>r_{b}\right)$, the system of nonstationary two-dimensional equations (1)-(4) reduces to a one-dimensional equation for the longitudinal electron flow velocity $V_{e z}$, and Eq. (14) can be approximated by

$$
-\frac{d}{r d r}\left[r\left(\frac{d p_{e z}}{d r}\right)\right]=\frac{4 \pi e^{2}}{c^{2}}\left(Z_{b} n_{b} V_{b}-n_{e} V_{e z}\right) .
$$

Note that Eq.(15) is valid in the nonlinear regime for arbitrary values of the plasma density. Equation (15) shows that degree of current neutralization is determined by the ratio of the beam radius $r_{b}$ to the skin depth $c / \omega_{p}$, similar to the what is found in linear theory [3]. If $r_{b}>c / \omega_{p}$, the ion current is well neutralized by the electron return current, i.e., the longitudinal electron velocity is reciprocal to plasma density $\left(V_{e z}=Z_{b} V_{b} n_{b} / n_{e}\right)$ and is small for plasma density much larger than the beam density. In the opposite limit $\left(r_{b}<<c / \omega_{p}\right)$, the ion beam current is not neutralized, and the electron longitudinal velocity is determined entirely by the ion beam current and does not depend on the plasma density.

The radial electron flow velocity may be determined from the electron continuity equation (1). We obtain

$$
V_{e r}=-\frac{1}{r n_{e}} \frac{\partial}{\partial \zeta} \int_{0}^{r}\left[n_{e}\left(V_{b}-V_{e z}\right)-n_{p} V_{b}\right] r d r
$$

where $n_{p}$ is the uniform background plasma density without the beam present. If quasineutrality is assumed, Eq. (16) simplifies to become

$$
V_{e r}=-\frac{1}{e r n_{e}} \frac{\partial}{\partial \zeta} \int_{0}^{r} j_{z} r d r
$$


where $j_{z}=e\left(n_{b} V_{b}-n_{e} V_{e z}\right)$ is the longitudinal current. The radial electron velocity is of order $\left(V_{b}-V_{e z}\right) r_{b} / l_{b}$, and is small compared with the beam velocity $V_{b}$. Substituting $V_{e r}$ and $V_{e z}$ into Eq.(7) then yields the electric field

$$
\mathbf{E}=-\frac{1}{e}\left(V_{b} \frac{\partial \mathbf{p}_{e}}{\partial \zeta}+\nabla K_{e}\right)
$$

Substituting $V_{e r}$ and $V_{e z}$ into Eq.(6) yields the azimuthal magnetic field

$$
B=-\frac{c}{e}\left(\frac{d p_{e z}}{d r}-\frac{d p_{e r}}{d z}\right)
$$

As can be seen from Eqs.(18) and (19), the values of electric and magnetic fields are strongly reduced in the case of dense plasma $\left(r_{b}>>c / \omega_{p}, n_{p}>>n_{b}\right)$ where the electron flow velocity is much smaller than beam velocity.

Finally, the degree of charge neutralization can be estimated directly from the Poisson's equation,

$$
\rho=\frac{1}{4 \pi} \nabla \cdot \mathbf{E}
$$

where $\rho=e\left(Z_{b} n_{b}-n_{e}\right)$. Using Eqs.(15), (18), and (20) it can be shown that the maximum deviation from quasineutrality, occurs when $r_{b} \sim c / \omega_{p}$, and $|\rho| \lesssim e \beta_{b}^{2} Z_{b} n_{b}$. Therefore, for nonrelativistic long ion pulses, $|\rho| / e Z_{b} n_{b}<<1$ and there is almost complete charge neutralization. For calculational purposes exact charge neutralization

$$
n_{e}=n_{b}+n_{p}
$$

can be assumed and deviations from quasineutrality can be calculated from Eq.(20) in a subsequent iteration. Section IV provides more quantitative estimates.

The radial force acting on the beam ions can also be determined in terms of the electron flow velocity. Substituting Eqs. (19) and (18) into the ion force equation yields

$$
F_{r}=e\left(E_{r}-\frac{1}{c} V_{b} B\right)=-\frac{\partial}{\partial r}\left(K_{e}-V_{b} p_{e z}\right)
$$

Because the radial flow velocity is small compared with the longitudinal flow velocity for long beams, it can be neglected in Eq. (22), which simplifies to become 


$$
F_{r}=\frac{\left(V_{b}-V_{e z}\right)}{\gamma_{e}} \frac{\partial}{\partial r} V_{e z}
$$

Equation (23) was derived in Ref. 8 in nonrelativistic form. Because $V_{e z}<V_{b}$, and because $V_{e z}$ is a monotonically decreasing function of radial coordinate $r$ (if $Z_{b} n_{b} V_{b}$ is a monotonically decreasing function of radial coordinate $r$ ), the radial force in Eq.(22) acting on the beam ions is always inward $\left(F_{r}<0\right)$, i.e., it pinches the ion pulse. The radial force in Eq.(22) is

greatly reduced for $V_{e z} \simeq V_{b}$, which corresponds to the case of good current neutralization, and background plasma density small compared to the beam density. The value of radial force acting on the beam ions is strongly reduced in the case of dense plasma $\left(r_{b}>>\right.$ $\left.c / \omega_{p}, n_{p}>>n_{b}\right)$ where the electron flow velocity is much smaller than beam velocity.

\section{EXAMPLES OF CALCULATIONS FOR HEAVY ION FUSION PARAMETERS}

We have performed self-consistent calculations of the electric and magnetic fields and the electron flow velocity according to Eqs. (13)-(16). In the first iteration, complete charge neutrality (21) is assumed. The corrections, including small departures from quasineutrality and the effects of displacement current and radial components of velocity [Eq.(14) compared to Eq. (15)], are then obtained in the second iteration. The electron velocity does not approach ultrarelativistic values even for $\beta_{b} \longrightarrow 1$, and therefore, ultrarelativistic effects are not important.

Typical results of the calculations are shown in Fig.1. The ion pulse is formed in the drift compression region of the accelerator and is assumed to have a density distribution corresponding to the self-similar solution in the drift compression region, i.e., a uniform ion density up to radius $r(z)=r_{b} \sqrt{1-\left(z / l_{b}\right)^{2}}$ [16], and zero density for larger radius [ Fig.1(a)]. For the conditions in Fig.1, the plasma density is chosen to be comparable to the ion beam density. The skin depth is assumed to be smaller than the beam radius, so the beam current is neutralized, and the longitudinal electron velocity is $V_{e z} \simeq V_{b} n_{b} /\left(n_{p}+n_{b}\right)$ in the beam region, and decays exponentially outside the beam over distances of order the skin depth 
[Fig. 1(b)]. The current is neutralized in the beam center up to about $80 \%$, and because the electron flow velocity is monotonically decreasing with radial coordinate $r$ [Fig.1(b)], the degree of current neutralization decreases towards the beam edge and approaches $50 \%$ at the boundary. Outside the beam, only the electron return current is present, and, therefore, the current is negative. [Fig.1(c)]. The longitudinal electric field $E_{z}$ is located mainly in front and in back of the beam to accelerate and decelerate electrons to the velocities required to assure that the electron return current neutralizes the ion beam current. Consequently, the longitudinal electric field $E_{z}$ is of order $m V_{e z}^{2} /\left(e l_{b}\right)$ [Fig.1(d)]. This electric field is small compared to the electric field of an unneutralized ion beam, and correspondingly the charge neutralization is close to unity, (typically about $98 \%$ in the head and tail of the beam in the regions of large gradients, and about $99.5 \%$ in the main body of the beam). The radial flow velocity calculated from Eq.(17) is depicted in Fig.1(e). As the beam enters the plasma, the integral $\int_{0}^{r} j_{z} r d r$ increases, and the radial flow velocity is negative, i.e., the beam attracts the background plasma electrons. Further from the beam head, the current neutralization is better, and $\int_{0}^{r} j_{z} r d r$ decreases and the radial flow velocity is positive. The radial electric field is calculated from the radial velocity in Eq.(18). Relativistic effects are not important for the conditions of Fig1, and the radial flow velocity is much smaller than the longitudinal velocity. Therefore, the radial electric field is determined approximately from $E_{r} \approx-m /(2 e)\left(\partial V_{e z}^{2} / \partial r\right)>0$, which is positive in the beam region [Fig.1(f)]. Similarly, from Eq.(19), the magnetic field is $B \approx-c m / e\left(\partial V_{e z} / \partial r\right)$, and is shown in Fig.1(g). Correspondingly, the longitudinal flow velocity is $V_{e z} \approx c E_{r} / B$. The situation is different in the radial direction, because the inertia and Lorentz force terms are comparable in size in the longitudinal projection of the momentum balance equation (2), and therefore $V_{e r} \neq c E_{z} / B$. The radial force acting on the beam ions is always negative as discussed above [Fig.1(h)].

Figure 2 depicts the longitudinal electron velocity and the electron streamlines for similar conditions to Fig.1, but for much smaller background plasma density. Under these conditions, the skin depth is much larger than the beam radius (5.6 times larger) outside the beam. Therefore, the electron velocity decays slowly outside the beam over distances of 
order the skin depth [Fig.2(a)]. Correspondingly, there is a sizeable radial electric field at distances much larger than the beam radius [Fig.2(b)]. Although the background plasma density is hundred of times smaller than the beam density, note that it effectively neutralizes both the beam current and charge. Because the electron longitudinal velocity is comparable in both cases [ $n_{p} \sim n_{b}$ in Fig1(b) and $n_{p}<<n_{b}$ in Fig. 2(a)], the electric fields are comparable for both cases and the degree of charge neutralization is similar.

In Fig. 3, the longitudinal electron flow velocity, azimuthal magnetic field, radial electric field, and radial force acting on the beam ions are shown at the midplane of the beam pulse for three values of plasma density. In a dense plasma $\left(n_{p}>>n_{b}\right)$, the electron velocity $\mathbf{V}_{e}$ is inversely proportional to the plasma density, in order to support the same return current and provide current neutrality. Therefore, the electric and magnetic fields also decrease with increasing plasma density. Figures 3(a) and 3(b) show that the radial force acting on the beam ions is less for small plasma density $\left(n_{p}<n_{b}\right)$, compared to the case where $n_{p} \sim n_{b}$. This is because the radial electric field nearly compensates the self-magnetic force $\left[V_{e z} \simeq V_{b}\right.$ in Eq.(22)]. For large plasma density $\left(n_{p}>>n_{b}\right)$, both the azimuthal magnetic field and the radial electric field are small due to the better current neutralization [Fig.3(c)]. During ballistic focusing, the beam reduces in radius by about a factor of ten, and the radial electric field and azimuthal magnetic field increase by a similar factor, which follows from Eq.(19).

Figure 4 shows the degree of fractional charge nonneutralization, $f=\rho /\left(e n_{b}\right)$, at the midplane of the beam for three values of the beam velocity. We have chosen parameters such that the departure from quasineutrality is approximately a maximum, corresponding to $r_{b}=c / \omega_{b}$, where $\omega_{b}^{2}=4 \pi e^{2} n_{b} / m_{e}$ is the electron plasma frequency calculated using the beam ion density. Moreover, small plasma density with $n_{p}=0.1 n_{b}$ is also assumed in Fig.4. For the conditions in Fig.4, the maximum value of $f$ is about $0.2 \beta_{b}^{2}$, which is much smaller than unity. Therefore, quasineutrality is very well satisfied.

To check the theoretical predictions, we developed a two-dimensional (2D) electromagnetic particle-in-cell (PIC) code [17]. In developing this PIC code, we followed the approach of Morse and Nelson [19]. The code uses a leap-frog, finite-difference scheme [18] to solve 
Maxwell's equations (3) and (4) on a two-dimensional rectangular grid in the frame moving with the beam. The current deposition scheme is designed to conserve charge exactly [19], so there is no need to solve Poisson's equation. Since the plasma ahead of the pulse is neutral, the boundary condition for the fields on the front boundary are trivial $(\mathbf{E}=\mathbf{B}=0)$. The dynamics of the (stationary) background ions is neglected, and the plasma electrons are treated as cold. The beam ions are represented by a stationary (in the moving frame) current density on the simulation grid. To advance the electrons, we use the time-centered leap-frog scheme first introduced in Ref. 19. In practice, the plasma is introduced at the front boundary, and difference equations are solved for several time steps in the laboratory frame. Then the electrons and fields are shifted backwards, and new plasma in introduced at the beam front. Periodic boundary conditions are used for the fields in the transverse direction. Electrons leaving through the side boundaries are reintroduced into the simulation box at the opposite sides. Electrons leaving the simulation box through the back boundary are removed from the simulation.

Figures 5 and 6 show the results of self-consistent electromagnetic two-dimensional particle-in-cell (PIC) simulations in slab geometry. The simulation results in Fig.5 show some phenomena associated with the finite neutralization length, neglected in the analytical theory, particularly the excitation of plasma waves by the beam front. The sharp ion beam front excites plasma waves. In linear theory, the plasma waves trail the beam front with period $l_{p}=2 \pi V_{b} / \omega_{p}$ and the elctron density is given by [4]

$$
n_{e}(\zeta, y)-n_{p}=\int_{-\infty}^{\zeta} \sin \left(\frac{\omega_{p}}{V_{b}}\left(\zeta-\zeta^{\prime}\right)\right) n_{b}\left(\zeta^{\prime}, y\right) \frac{\omega_{p}}{V_{b}} d \zeta^{\prime}
$$

Assuming a step function profile for $n_{b}\left(\zeta^{\prime}, y\right)$, linear theory predicts electron density oscillations with amplitude $n_{b}$ inside the beam, i.e.,

$$
n_{e}(z, y)-n_{p}=\left[1-\cos \left(\frac{\omega_{p}}{V_{b}}\left(z_{b}(y)-z\right)\right)\right] n_{b},
$$

where $z_{b}(y)$ is the coordinate of beam front. Figure 5 (a) shows that the linear results are not valid, and the amplitude of oscillations can be as much as six times larger than the linear 
results. Nonlinear effects can also account for large increases in wave amplitude [21], even in a cold plasma model. Figure 5 also shows the importance of two-dimensional effects. The linear result in Eq.(24) predicts that the plasma waves trail the beam front independently for any given $y$, and should not decay. Clearly, linear theory cannot predict the features of the plasma waves in Fig.5, even qualitatively. The plasma waves in Fig.5 have a twodimensional structure. Furthermore, the plasma waves are excited before the beam front at the radial edge of the beam [see Fig.5(c)] and decay away from the front, in contrast to the predictions of linear theory. The accurate description of plasma waves has to be performed using a nonlinear two-dimensional model and is beyond the scope of present paper.

To minimize the excitation of plasma waves, a smooth ion beam profile is used in the simulations shown in Fig.6. We choose the profile

$$
n_{b}(y, z)=f\left(\sqrt{\left(\frac{z}{l_{b}}\right)^{2}+\left(\frac{y}{r_{b}}\right)^{2}}\right),
$$

where

$$
f(s)=\left\{\begin{array}{cc}
0, & s>1 \\
g\left(\frac{1-s}{a}\right), & 1-a<s<1 \\
1, & s<1-a .
\end{array}\right\}
$$

Here, $g(t)=10 t^{3}-15 t^{4}+6 t^{5}$, and $a$ is a parameter characterizing the width of profile smoothing. Plasma waves are not excited if the width of the beam front is much longer than the plasma period, i.e., $a l_{b}>>l_{p}$. For example, plasma waves are very weakly excited for the conditions chosen in Fig.6, where $a l_{b}=6 l_{p} / \pi$, and the electron density is equal to the ion density within noise errors [Fig.6(a)].

Figure 6 shows good agreement between results of the PIC simulations and the fluid calculations, both performed in slab geometry. The establishment of quasineutrality is clearly evident in Fig.6(a). Small deviations from quasineutrality are due to numerical noise and excitation of plasma waves by the beam front. Figure 6(b) shows the difference in the current profile at two different beam cross-sections. In the region of the beam head $(z=$ 
$\left.25 c / \omega_{p}\right)$ the beam radius $r_{b}=1.6 c / \omega_{p}$ is comparable to the skin depth, and correspondingly the electron current neutralizes about $80 \%$ of the ion current. In the midplane of the beam $(z=0)$, the beam radius $r_{b}=3 c / \omega_{p}$ is larger than the skin depth, and correspondingly the electron current neutralizes more than $90 \%$ of the ion current in the beam center. The degree of current neutralization is smaller at the beam edge due to the sharp variation of the ion current profile. According to Eq.(15), the electron return current is a smooth decreasing function of radial coordinate $r$, and cannot neutralize (the nearly discontinuous) ion current. The magnetic field shows very good agreement between the analytic formulas [Eqs.(15) and (19)] and the PIC simulation results. The amplitude of the electric field is much smaller than the magnetic field. Therefore, the contributions due to numerical noise and plasma waves are more pronounced in Fig.6 (d) and Fig.6 (e) compared with Fig.6 (c). For the conditions in Fig.6, the spatial resolution was $932 \times 198$ with 9 particles per cell, which totals more than one and one-half million particles and requires a few hours of calculations on a one-processor Dell Pentium $1 G H z$ workstation. it is evident that an accurate calculation of the electric field using PIC simulations is very cumbersome in contrast with the semianalytical approach described above. In summary, the very good agreement of the electric and magnetic fields validates the proposed theory.

\section{CONCLUSIONS AND OUTLOOK}

The propagation of a finite-length ion beam pulse through a uniform, stationary background plasma has been studied. The analytical solutions for the electric and magnetic fields generated by an ion beam pulse have been determined in the nonlinear case for arbitrary values of $n_{p} / n_{b}$ under the assumption of a long beam, where the beam length is much longer than the beam radius and the plasma neutralization length $\left(V_{b} / \omega_{b}\right)$. Under these conditions, a reduction in the dimensionality of the problem is possible. Assuming an axisymmetric beam, the longitudinal electron flow velocity is determined for one-dimensional variations in the radial direction for each axial slice of the beam. The electric and magnetic 
fields are then readily calculated from the longitudinal electron flow velocity. As a result, numerical simulations are very fast, even for very long beams with a large ratio of the beam length to the beam radius. Since the electron response time is much faster than the ion beam pulse duration, any variations in plasma or beam parameters are adiabatically slow on the electron time scale. The approach used here can be generalized to the case of nonuniform plasma density and beam density profiles, and forms the basis for a hybrid semi-analytical approach to be used for calculations of beam propagation in the target chamber. This work is now underway.

The assumption of zero generalized vorticity can be broken by plasma generation inside the beam. Therefore, if a considerable amount of plasma is produced by beam ionization processes, the approach presented here requires modification.

The assumption of immobile background ions can be incorrect for very long beams. Indeed, the radial displacement of a plasma ion with mass $m_{i}$ during the beam pulse duration $2 l_{b} / V_{b}$ caused by the radial electric field in Eq.(18) [which at maximum is of order $m V_{b}^{2} /\left(e r_{b}\right)$ ] is larger than the beam radius whenever $\left(m / m_{i}\right)\left(2 l_{b} / r_{b}\right)^{2}>1$. For the beams parameters considered for heavy ion fusion, the beam aspect ratio $\left(l_{b} / r_{b}\right)$ is less than one hundred, and the background ion dynamics can be neglected for all gases, except possibly for hydrogen.

The analytical formulas derived in this paper can provide an important benchmark for numerical codes and provide scaling laws for different beam and plasma parameters. The simulations of current and charge neutralization performed for conditions relevant to heavy ion fusion typically showed very good charge and current neutralization. Moreover, an important conclusion of the present analysis is that for long, dense beams (length $l_{b}>>V_{b} / \omega_{b}$, where $V_{b}$ is the beam velocity, and $\omega_{b}$ is the electron plasma frequency evaluated with the ion beam density), the charge neutralization is very good even for a tenuous background plasma with density much smaller than the beam density. The background plasma is collected radially over the distances of order the electron skin depth, and the small value of plasma density can be well compensated by the large dimension of the collecting area.

Acknowledgment This research was supported by the U.S. Department of Energy 
Office of Fusion Energy Sciences and Division of High Energy Physics. It is a pleasure to acknowledge the benefit of useful discussions with Ed Lee, Marshall Rosenbluth and Wei-li Lee. 


\section{Figure captions}

Fig.1. Characteristics of the ion beam pulse are: singly-charged $C s^{+}$ions; ion energy $E_{b}=4 G e V$; maximum ( in the middle section of the beam) ion current $I_{b}=4 k A$; maximum beam radius $r_{b}=3 \mathrm{~cm}$; half-length $l_{b}=40 \mathrm{~cm}$; and background plasma density $n_{p}=10^{11} \mathrm{~cm}^{-3}$. Shown in the figure are contour plots in $\left(z / l_{b}, r / r_{b}\right)$ space of: (a) ion density $n_{b}$ in the beam pulse; (b) normalized longitudinal electron velocity $V_{e z} / V_{b}$; (c) normalized electron current $1-n_{e} V_{e z} /\left(N_{b} V_{b}\right)$, where $N_{b} \equiv n_{b}(0,0)$; (d) normalized longitudinal electric field $E_{z} / E_{z 0}$, where $E_{z 0} \equiv m V_{b}^{2} /\left(e l_{b}\right)=410 \mathrm{~V} / \mathrm{cm}$; (e) normalized radial electron velocity $V_{e r} l_{b} / r_{b} V_{b}$; (f) normalized radial electric field in the beam $E_{r} / E_{r 0}$, where $E_{r 0} \equiv m V_{b}^{2} /\left(e r_{b}\right)=5.467 \mathrm{kV} / \mathrm{cm} ;(\mathrm{g})$ normalized azimuthal magnetic field $B / B_{0}$, where $B_{0} \equiv m c V_{b} /\left(e r_{b}\right)=74 G$; and $(\mathrm{h})$ normalized radial force acting on the beam ions $\left(E_{r}-\beta_{b} B\right) / E_{r 0}$, where $E_{r 0} \equiv m V_{b}^{2} /\left(e r_{b}\right)=5.467 k V / c m$.

Fig.2. Characteristics of the ion beam pulse are the same as in Fig.1 but the background plasma density is $n_{p}=10^{9} \mathrm{~cm}^{-3}$. Shown in the figure are contour plots in $\left(z / l_{b}, r / r_{b}\right)$ space of: (a) normalized longitudinal electron velocity $V_{e z} / V_{b}$; and (b) normalized integrated radial flux of electrons $\Gamma_{z}=\int_{0}^{r}\left[n_{e}\left(V_{b}-V_{e z}\right)-n_{p} V_{b}\right] r d r$. The contour plots of $\Gamma_{z}$ coincide with the electron trajectories in a frame moving with the beam.

Fig.3. Radial plots of the normalized electron velocity $\left(V_{e z} / V_{b}\right)$, the radial electric field in the beam $\left(E_{z} / E_{r 0}\right)$, the azimuthal magnetic field in the beam $\left(B / B_{0}\right)$, and the radial force acting on the beam ions $\left.\left(E_{r}-\beta_{b} B\right) / E_{r 0}\right)$, shown in the midplane of the ion pulse for the same conditions as in Fig.1. The three plots correspond to plasma densities: (a) $n_{p}=0.310^{11} \mathrm{~cm}^{-3}$, (b) $n_{p}=10^{11} \mathrm{~cm}^{-3}$, (c) $n_{p}=10^{12} \mathrm{~cm}^{-3}$.

Fig.4. Radial plots of the degree of fractional charge nonneutralization $f=\rho /\left(e n_{b}\right)$ at the midplane of the ion beam pulse in background plasma assuming three values of beam velocity $\beta_{b} c$. The beam radius is chosen to be $r_{b}=c / \omega_{b}$, where $\omega_{b}^{2}=4 \pi e^{2} n_{b} / m_{e}$ is the electron plasma frequency calculated using the beam ion density. The beam radius chosen in the figure corresponds to the maximum degree of fractional charge nonneutralization. 
The corresponding beam current is $4.25 \beta_{b} k A$, and the plasma density is $n_{p}=0.1 n_{b}$.

Fig.5. The excitation of plasma waves by the beam head is calculated in two-dimensional slab geometry using the PIC-MC code [17] for the following dimensionless beam parameters: $\beta_{b}=0.5, r_{b}=1.5 c / \omega_{p}, l_{b}=15 c / \omega_{p}, n_{p}=n_{b}$, and $Z_{b}=1$. (a) Shown in the figure are electron charge density contour plots in $\left(\omega_{p} z / c, \omega_{p} y / c\right)$ parameter space, and . electron charge density versus $\left(\omega_{p} z / c\right)$ (b) for $y=0$ and (c) for $y=c / \omega_{p}$ arrows show beam edge.

Fig.6. Comparison of 2D particle-in-cell simulations with theoretical predictions for the following dimensionless parameters: $\beta_{b}=0.5, r_{b}=1.5 c / \omega_{p}, l_{b}=30 c / \omega_{p}$, and $n_{p}=n_{b}$ performed in two beam cross-sections: the midplane at $z=0$, and near the beam front at $z=25 c / \omega_{p}$. The ion profile is smoothed according to Eq.(26), with $a l_{b}=6 c / \omega_{p}=$ $(6 / \pi) l_{p}$. Shown are radial plots of (a) normalized electron density $n_{e} / n_{p} ;$ (b) normalized longitudinal current $\left(n_{b} V_{b}-n_{e} V_{e z}\right) /\left(n_{p} c\right)$; (c) normalized azimuthal magnetic field, $e B /\left(2 m \omega_{p} c\right)$; (d) the normalized radial electric field $e E_{r} /\left(2 m \omega_{p} c\right)$; and (e) normalized longitudinal electric field $e E_{z} /\left(2 m \omega_{p} c\right)$. 


\section{REFERENCES}

[1] H. Alfven, Phys. Rev. 55, 425 (1939).

[2] W. H. Bennett, Phys. Rev. 45, 890 (1934).

[3] D. A. Hammer and N. Rostoker, Phys. Fluids 13, 1831 (1970); J. L. Cox, and W.H. Bennett, Phys. Fluids 13, 182 (1970).

[4] P. Chen, J.M. Dawson, R. W. Huff, and T. Katsouleas, Phys. Rev. Lett. 51, 693 (1985).

[5] P. Chen, Part. Accel. 20, 171 (1987); P. Chen, J.J. Su, T. Katsouleas, S. Qilks, and J. M. Dawson, IEEE Trans. on Plasma Science PS.-15, 218 (1987).

[6] R. Govil, W.P. Leemans, E. Yu. Backhaus and J.S. Wurtele, Phys. Rev. Lett. 83, 3202 (1999); G. Hairapetian, P. Davis, C.E. Clayton, C. Joshi, S.C. Hartman, C. Pellegrini, T. Katsouleas, Phys. Rev. Lett. 72, 2403 (1994).

[7] M. Roth, T.E. Cowan, M.H. Key, S.P. Hatchett, et al., Phys. Rev. Lett. 86, 436 (2001); M. Tabak, J. Hammer, M. E. Glinsky, W. L. Kruer, S. C. Wilks, J. Woodworth, E. M. Campbell, M. D. Perry, and R. J. Mason, Phys. Plasmas 1, 1626 (1994).

[8] K.Hahn and E. P. Lee, J. Fusion Engineering and Design 32-33, 417 (1996).

[9] S. Rajagopalan, D.B. Cline , P. Chen, Nucl. Instrum. Meth.A 355, 169 (1995); J.S.T. Ng, P. Chen, H. A. Baldis, P. Bolton, etal., "Results on Plasma focusing of high energy density electron and positron beams", Invited talk presented at the 9th Workshop on Advanced Accelerator Concepts, Santa Fe, New Mexico, USA, June11-16, 2000; SLACPUB-8565, Aug 2000.

[10] T. Tauschwitz, S.S. Yu, S. Eylon, L. Reginato, W. Leemans, J.O. Rasmussen, R.O. Bangerter J. Fusion Engineering and Design 32-33, 493 (1996)

[11] B.N. Breizman, , D.L. Fisher, P.Z. Chebotaev, T. Tajima, Excitation of Nonlinear Wake Field in a Plasma for Particle Acceleration, in Research Trends in Physics: Coherent 
Radiation Generation and Particle Acceleration, edited by A. M. Prokhorov (American Institute of Physics, New York, 1992), p. 263, and IFS report \#502 (1992); J.B. Rozenzweig, D.B. Cline, H. Figueroa, W. Gai, R. Wonecny, J Norem, P. Schoessow, and G. Simpson, Phys. Rev. Lett. 61, 98 (1988); J.B. Rosenzweig, B.N. Breizman, T. Katsouleas, and J.J. Su, Phys. Rev. A 44, R6189 (1991).

[12] M. Rosenbluth, E.P. Lee and R. Briggs, Proceedings of the International Symposium on Heavy Ion Fusion, San Diego (2000).

[13] W. Lee, H. Qin, R. Davidson, Proceedings of the International Symposium on Heavy Ion Fusion, San Diego (2000).

[14] L.M. Gorbunov, Sov. Phys. Usp.16, 217 (1973).

[15] E. P. Lee, S.Yu, H.L. Buchanan, F.W. Chambers, M.N. Rosenbluth, Phys. Fluids, 23, $2095(1980)$

[16] D.D.-M. Ho, S.T. Brandon and E.P. Lee, Part. Accel. 35, 15 (1991).

[17] E. A. Startsev and C. J. McKinstrie, "Particle-in-cell simulations of ponderomotive particle acceleration in a plasma" submitted to Phys. Rev. E (2000).

[18] C.K. Birdsall and A. B. Langdon, Plasma Physics via Computer Simulations, 2nd Ed., (Plenum, New York, 1984).

[19] R. L. Morse and C. W. Nielson, Phys. Fluids 14, 830 (1970).

[20] J. P. Boris and D. A. Hammer, Phys. Rev. E 10, 3338 (1969).

[21] R. C. Davidson, Methods in Nonlinear Plasma Theory (New York, Academic Press, 1972) p.39 . 

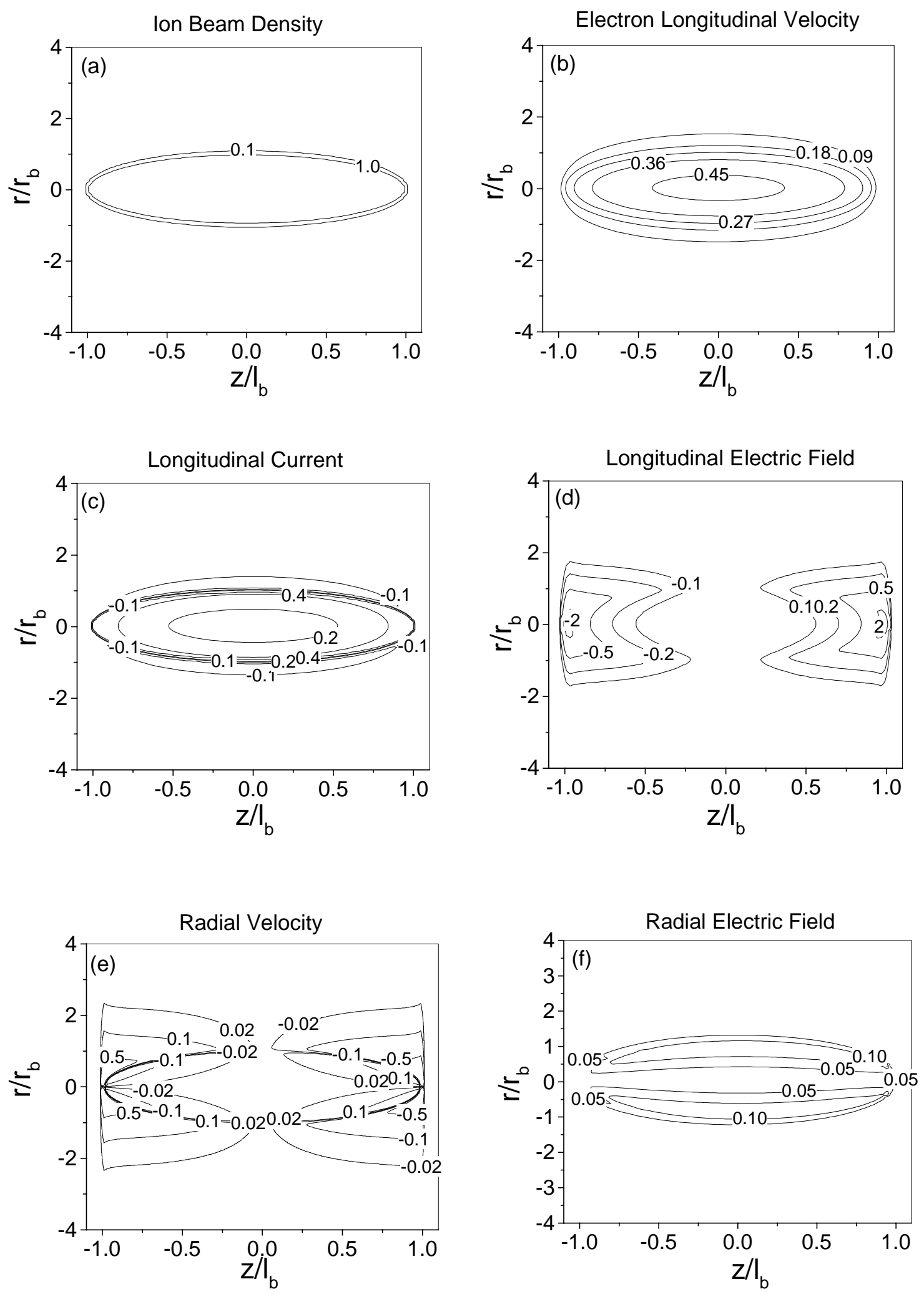

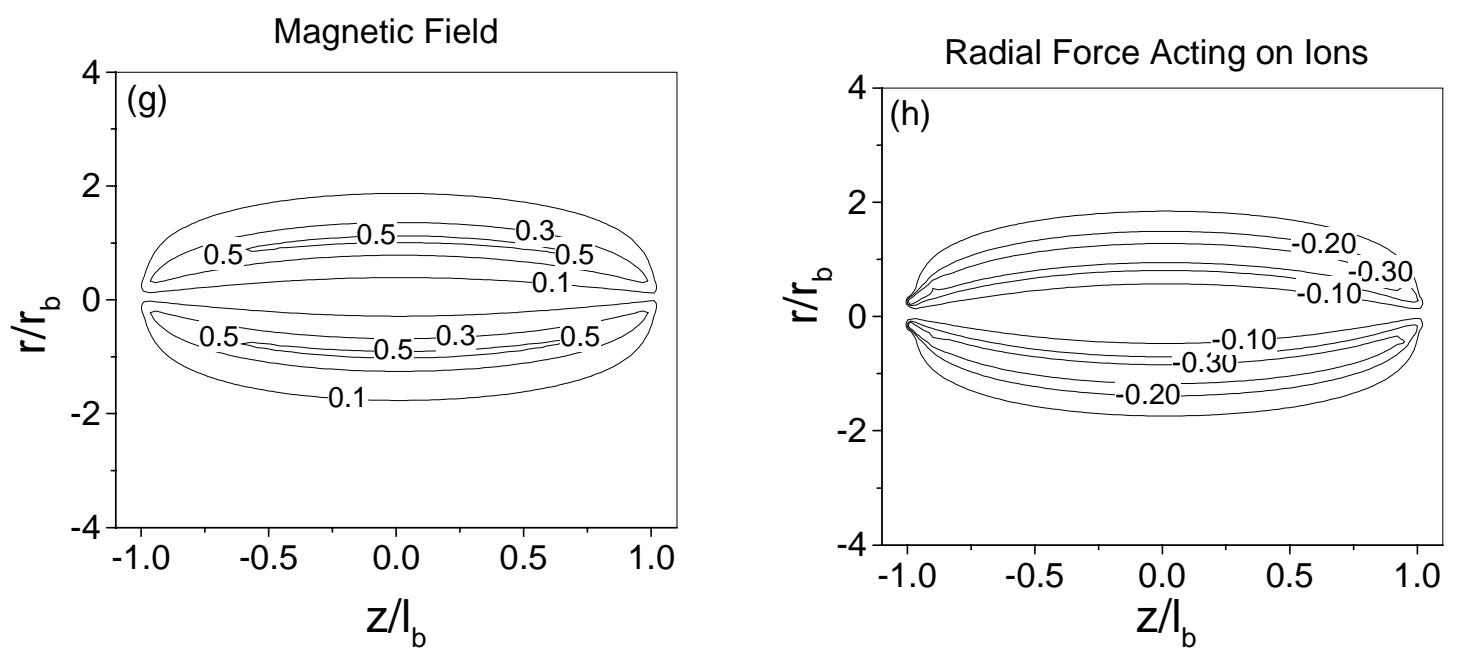

1

\footnotetext{
${ }^{1}$ Fig.1 Kaganovich et al
} 

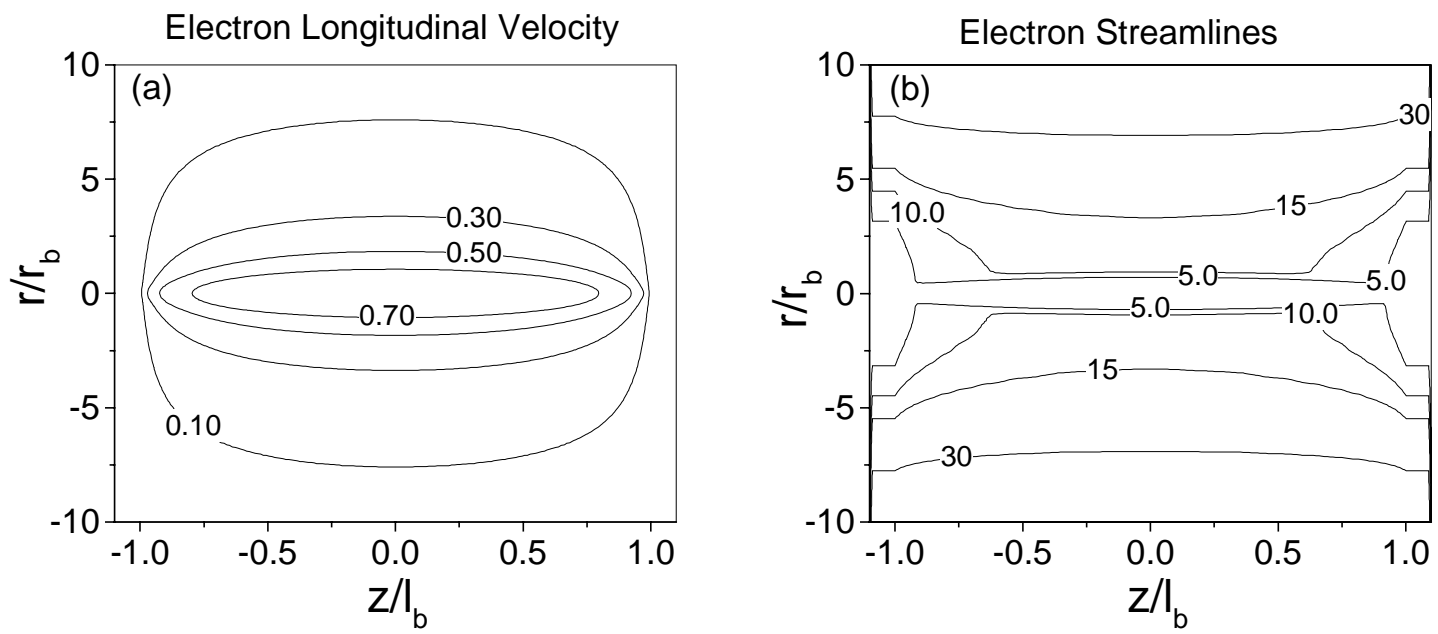

2

\footnotetext{
${ }^{2}$ Fig.2 I. Kaganovich et al
} 

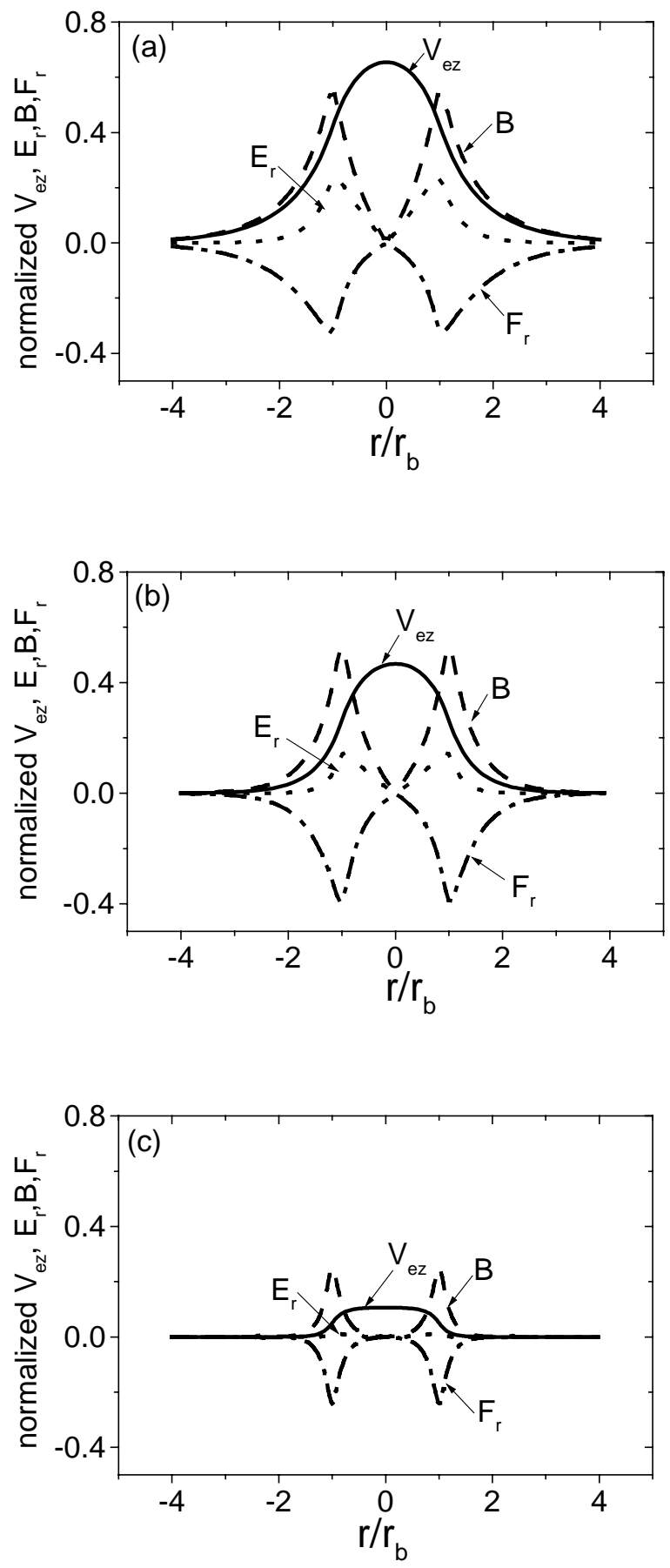

\footnotetext{
${ }^{3}$ Fig.3 I. Kaganovich et al
} 


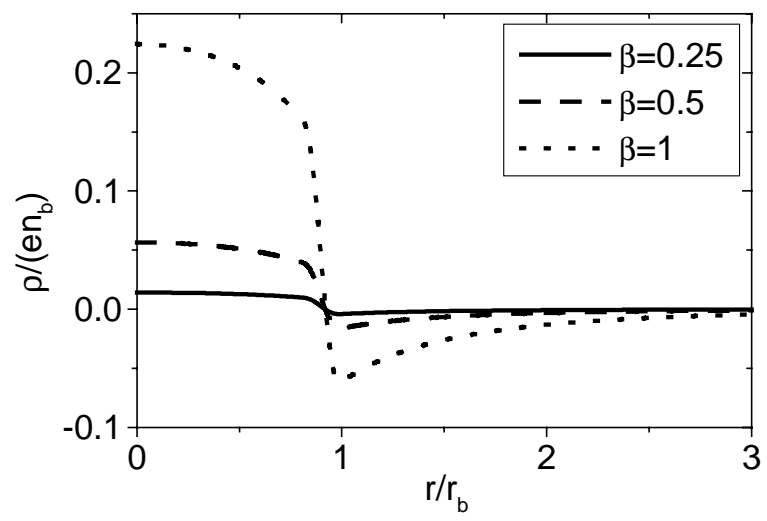

1

\footnotetext{
${ }^{1}$ Fig. 4 Kaganovich I.
} 


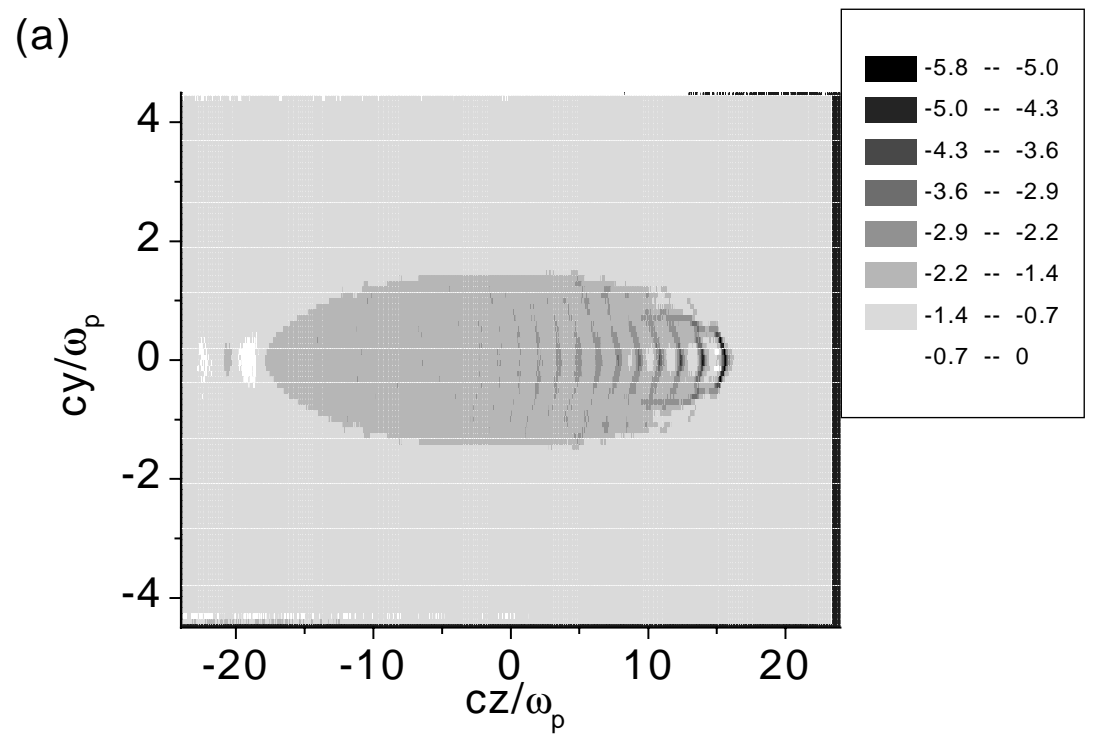

(b)

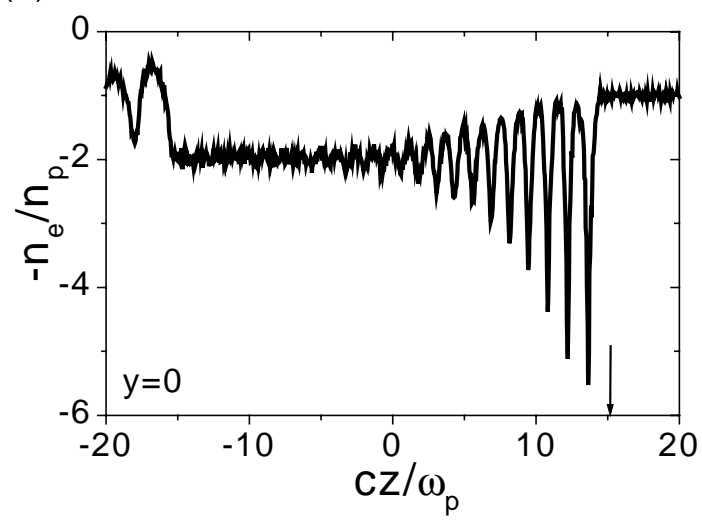

(c)

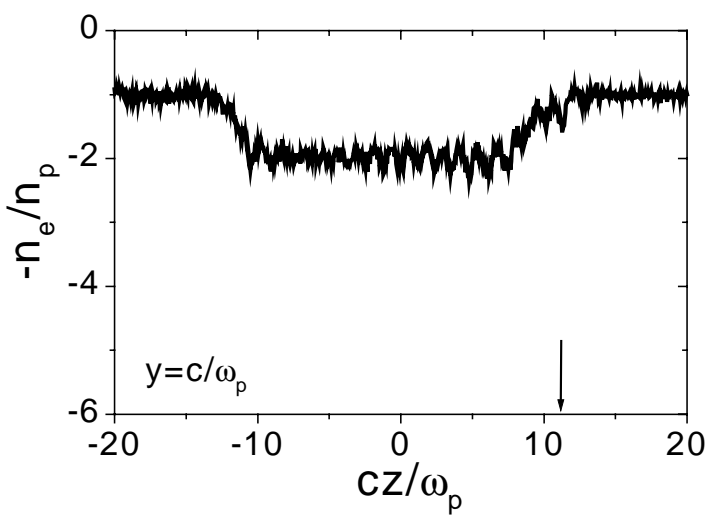



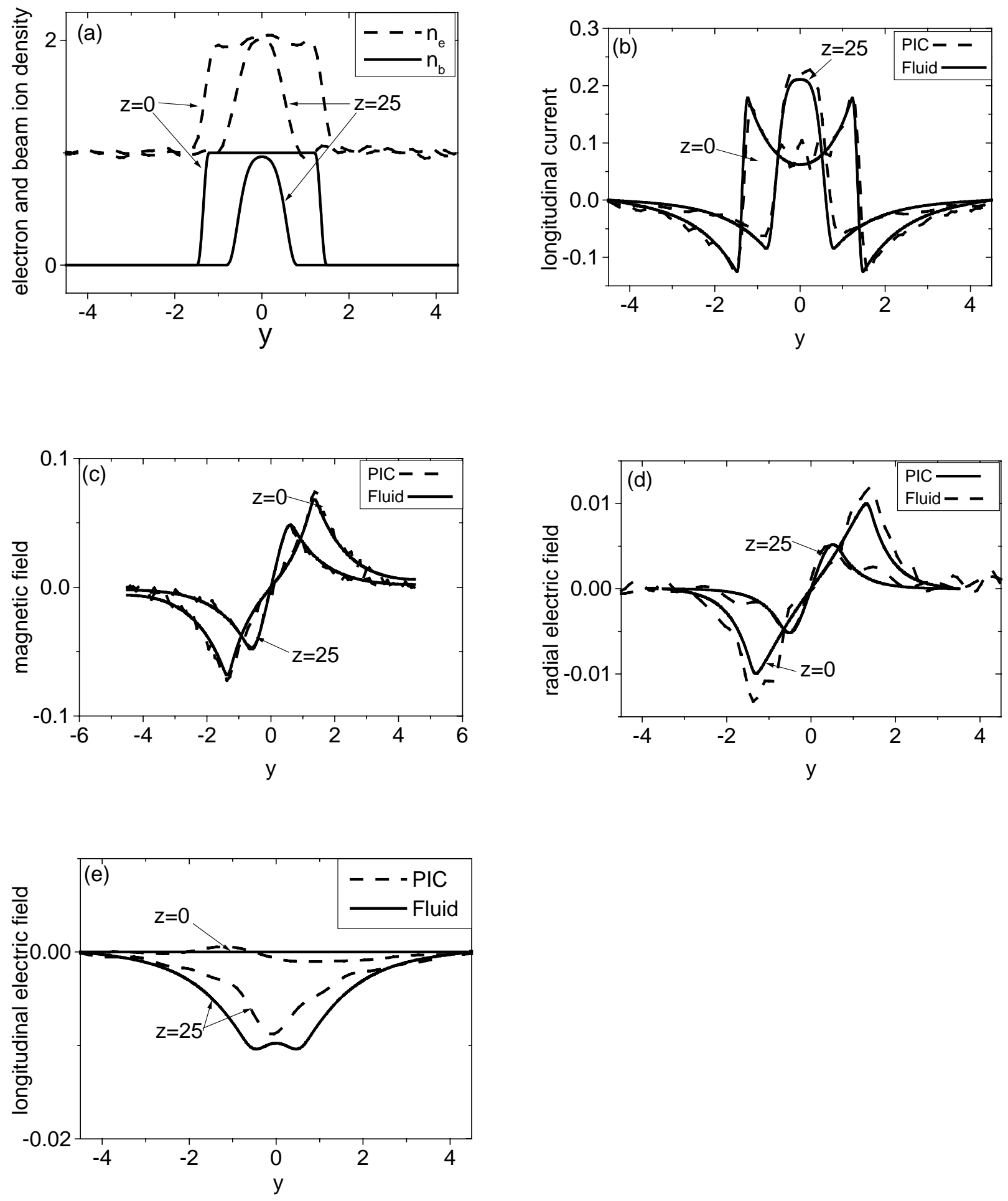


\section{External Distribution}

Plasma Research Laboratory, Australian National University, Australia

Professor I.R. J ones, Flinders University, Australia

Professor J oão Canalle, Instituto de Fisica DEQ/IF - UERJ , Brazil

Mr. Gerson O. Ludwig, Instituto Nacional de Pesquisas, Brazil

Dr. P.H. Sakanaka, Instituto Fisica, Brazil

The Librarian, Culham Laboratory, England

Library, R61, Rutherford Appleton Laboratory, England

Mrs. S.A. Hutchinson, JET Library, England

Professor M.N. Bussac, Ecole Polytechnique, France

Librarian, Max-Planck-Institut für Plasmaphysik, Germany

J olan Moldvai, Reports Library, MTA KFKI-ATKI, Hungary

Dr. P. Kaw, Institute for Plasma Research, India

Ms. P.J . Pathak, Librarian, Insitute for Plasma Research, India

Ms. Clelia De Palo, Associazione EURATOM-ENEA, I taly

Dr. G. Grosso, Instituto di Fisica del Plasma, Italy

Librarian, Naka Fusion Research Establishment, J AERI, J apan

Library, Plasma Physics Laboratory, Kyoto University, J apan

Research Information Center, National Institute for Fusion Science, J apan

Dr. O. Mitarai, Kyushu Tokai University, J apan

Library, Academia Sinica, Institute of Plasma Physics, People's Republic of China

Shih-Tung Tsai, Institute of Physics, Chinese Academy of Sciences, People's Republic of China

Dr. S. Mirnov, Triniti, Troitsk, Russian Federation, Russia

Dr. V.S. Strelkov, Kurchatov Institute, Russian Federation, Russia

Professor Peter Lukac, Katedra Fyziky Plazmy MFF UK, Mlynska dolina F-2, Komenskeho Univerzita, SK-842 15 Bratislava, Slovakia

Dr. G.S. Lee, Korea Basic Science Institute, South Korea

Mr. Dennis Bruggink, Fusion Library, University of Wisconsin, USA

Institute for Plasma Research, University of Maryland, USA

Librarian, Fusion Energy Division, Oak Ridge National Laboratory, USA

Librarian, Institute of Fusion Studies, University of Texas, USA

Librarian, Magnetic Fusion Program, Lawrence Livermore National Laboratory, USA

Library, General Atomics, USA

Plasma Physics Group, Fusion Energy Research Program, University of California at San Diego, USA

Plasma Physics Library, Columbia University, USA

Alkesh Punjabi, Center for Fusion Research and Training, Hampton University, USA

Dr. W.M. Stacey, Fusion Research Center, Georgia Institute of Technology, USA

Dr. J ohn Willis, U.S. Department of Energy, Office of Fusion Energy Sciences, USA

Mr. Paul H. Wright, Indianapolis, Indiana, USA 
The Princeton Plasma Physics Laboratory is operated by Princeton University under contract with the U.S. Department of Energy.

\author{
Information Services \\ Princeton Plasma Physics Laboratory \\ P.O. Box 451 \\ Princeton, NJ 08543
}

Phone: 609-243-2750

Fax: 609-243-2751

e-mail: pppl_info@pppl.gov

Internet Address: http://www.pppl.gov 